\title{
tuberías de materiales plásticos para agua caliente
}

Centre d'Asisstance Technique et de Documentation du Bâtiment et des Travaux Publics.

(C. A. T. E. D.)

Nota Técnica 98. Noviembre 1967

Los tubos de material plástico, aunque relativamente recientes, se emplean ahora mucho en la construcción. Las variedades más corrientes son el cloruro de polivinilo (P.V.C.) (1) y el polietileno. Sin embargo, estos materiales resisten bastante mal el calor. lo que excluye su empleo para el transporta de agua caliente.

Esta nota reúne algunas indicaciones sobre ciertos materiales plásticos que resisten mejor el calor.

Hemos limitado este estudio a algunas variedades, cuyos precios parecen relativamente interesantes; se trata, principalmente:

- del cloruro de polivinilo perclorado (P.V.C.C.);

- del acrilonitrilo butadieno estireno (A.B.S.);

- del polipropileno (P.P.);

— de las poliamidas (P.A.);

- de los termoendurecibles estratificados (2).

Estas tuberías se han empleado hasta la fecha para instalaciones de fluidos calientes, en particular en la industria o en los laboratorios.

Para emplearlos en distribución de agua caliente sanitaria, la experiencia sobre estos materiales es todavía muy limitada; son necesarias todavía numerosas puestas a punto para obtener soluciones durables.

(1) Las tuberías en cloruro de polivinilo (P.V.C.) son objeto de reglas muy precisas en cuanto a su utilización: alimentación en agua fría (DTU 60.31) y descendentes de agua de lluvia (DTU 60.33) Para la distribución de agua fría y las descendentes de agua de lluvia, las instalaciones de tubos en P.V.C. están cubiertas por la policía "individual de base" bajo las siguientes reservas: - los tubos y racores deben llevar la marca de calidad PF expedida por el Centro de Estudio de Materiales Plásticos;

- la puesta en obra debe hacerse conforme a DTU 60.31 y DTU 60.32. Las tuberías de plástico distintas del P.V.C. no se incluyen en la garantía decenal, así como tampoco las tuberias del P.V.C. empleadas para la evacuación de aguas residuales.

(2) Los tubos en termoendurecedores estratificados no existen en diámetros inferiores a $40 \mathrm{~mm}$. 
Se insiste sobre el hecho de que las instalaciones realizadas con estos tubos no están cubiertas por la policía de seguro "individual de base" y que deben ser objeto:

- bien de una descarga de responsabilidad por parte del cliente;

- bien de una garantía del suministrador que cubra la sustitución de los tubos, la mano de obra y los perjuicios eventuales.

\section{VENTAJAS DEL TUBO DE PLASTICO}

\section{Resistencia a la corrosión}

Los materiales plásticos resisten bien, generalmente, la acción de productos químicos, aunque mantengan cierta sensibilidad a los disolventes y a ciertos ácidos concentrados (los fabricantes suministran en general una tabla de resistencias químicas). Presentan la ventaja esencial sobre los tubos de acero de no ser atacados por el agua potable que se distribuye normalmente.

\section{Incrustaciones}

Para las velocidades habituales de circulación (superiores a $0,6 \mathrm{~m} / \mathrm{s}$ ), la formación de incrustaciones es prácticamente nula.

\section{Pérdida de carga}

Las superficies internas de las tuberías permanecen mucho más lisas que las de las tuberías metálicas, lo que permitiría eventualmente reducir los diámetros de 10 a $15 \%$ (3).

\section{Puesta en obra}

La puesta en obra es relativamente simple cuando los empalmes pueden hacerse por pegado. La ligereza del material facilita mucho la puesta en obra.

\section{Aislamiento térmico}

Los materiales plásticos son mucho más aislantes que los metales; pero no deben de hacerse ilusiones ya que, siendo los espesores pequeños, las pérdidas de calor sólo se disminuyen ligeramente.

\section{Aislamiento acústico}

La transmisión de las vibraciones por los tubos plásticos es más débil que con las tuberías metálicas; pero si la flexibilidad del material permite absorber parte de las vibraciones, la transmisión se hace aún por el agua; además, si son delgadas, las tuberías permanecen sonoras.

(3) Esta posibilidad no está admitida oficialmente. El DTU no habla de ella. 


\section{INCONVENIENTES DEL TUBO DE PLASTICO}

\section{Resistencia al calor}

Cualquiera que sea su origen, los materiales plásticos resisten siempre difícilmente al calor. Es el mayor inconveniente que presentan con relación a los tubos metálicos.

Las resistencias a la tracción disminuyen mucho cuando la temperatura aumenta; cuanto más elevada es la temperatura del agua, más se debe limitar la presión en la red.

Para una misma familia de materiales plásticos es difícil establecer una curva media de resistencia, puesto que, según las fórmulas, las características varían entre amplios márgenes (fig. 1).

Fig. 1.-Tensión de rotura instantánea en función de la temperatura (las dos curvas dadas para cada familia representan dos de las variedades más corrientes).

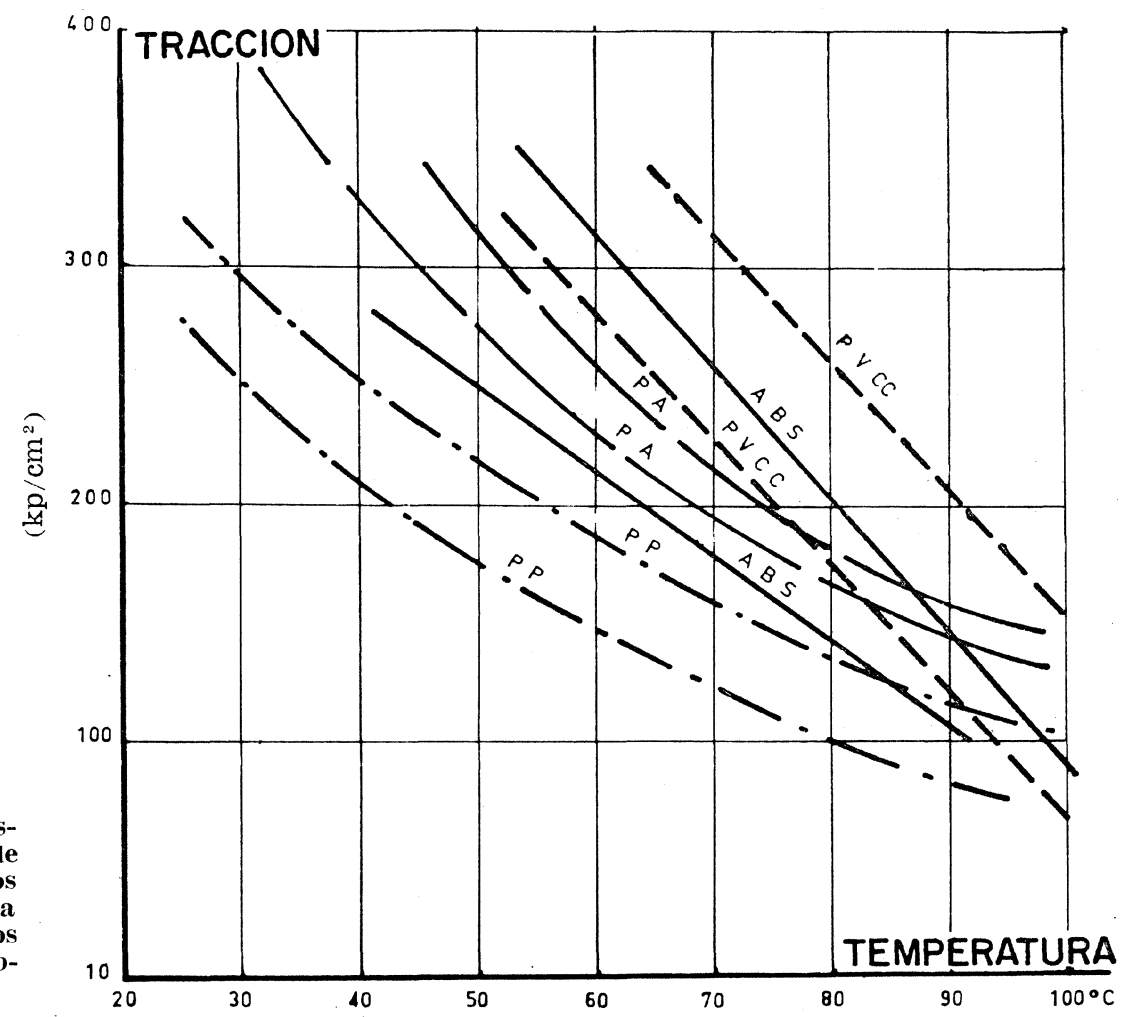

Se destaca que entre $20^{\circ}$ y $100^{\circ} \mathrm{C}$, la caída de resistencia es generalmente superior a $60 \%$. Estas medidas corresponden a experiencias de corta duración. De hecho, los materiales plásticos sometidos a tensiones continuas sufren una fluencia tanto más importante cuanto más elevada es la temperatura.

Para estimar la resistencia de un material plástico, es preciso tener en cuenta la evolución de las características en el tiempo (se trata de medidas de envejecimiento). 


\section{Envejecimiento}

Es la característica peor conocida de los nuevos materiales plásticos. A la fluencia bajo carga, citada anteriormente, se añaden diversos efectos que fatigan el material; se trata de:

- alternancia de las variaciones de tensiones debida a la presión y a la temperatura;

- acción de los rayos ultravioletas;

- reacciones químicas en contacto con diversos productos: ácidos, disolventes, producios clorados, oxigeno del aire;

- absorción de agua.

Las experiencias han permitido medir la tensión de rotura, teniendo en cuenta un cierto envejecimiento, para el P.V.C.C. (fig. 2), para el polipropileno (fig. 3), para el A.B.S. (fig. 4), y para la poliamida (fig. 5).
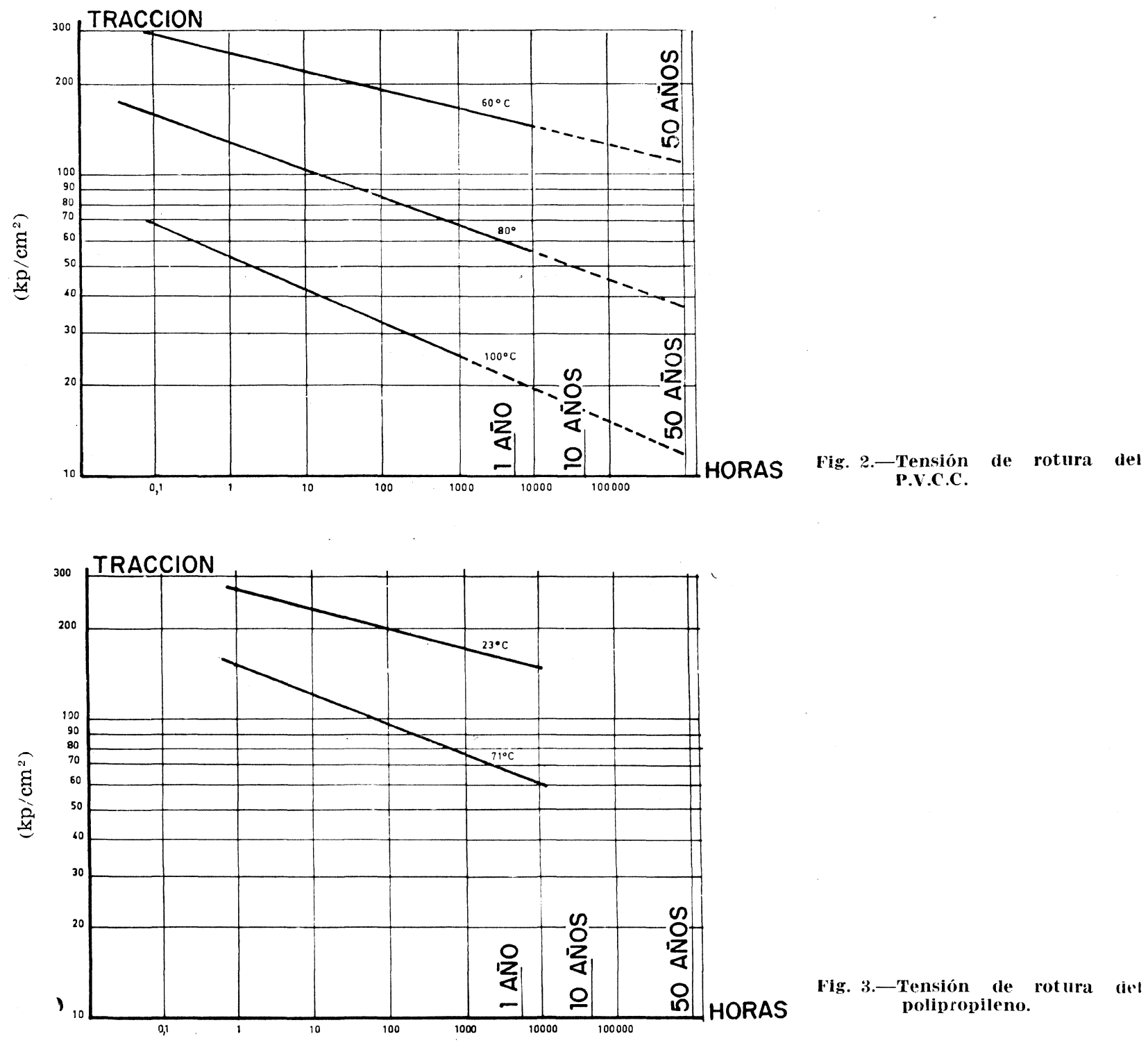
Fig. 4.-Tensión de rotura

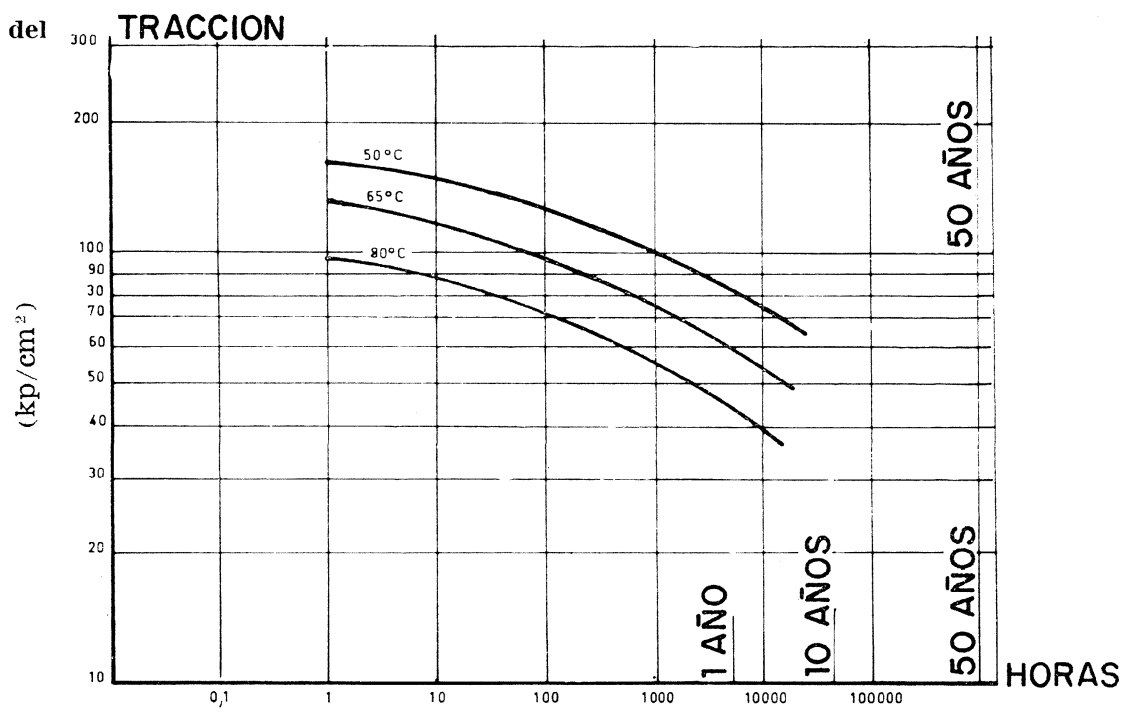

Fig. 5.-Tensión de rotura de la poliamida.

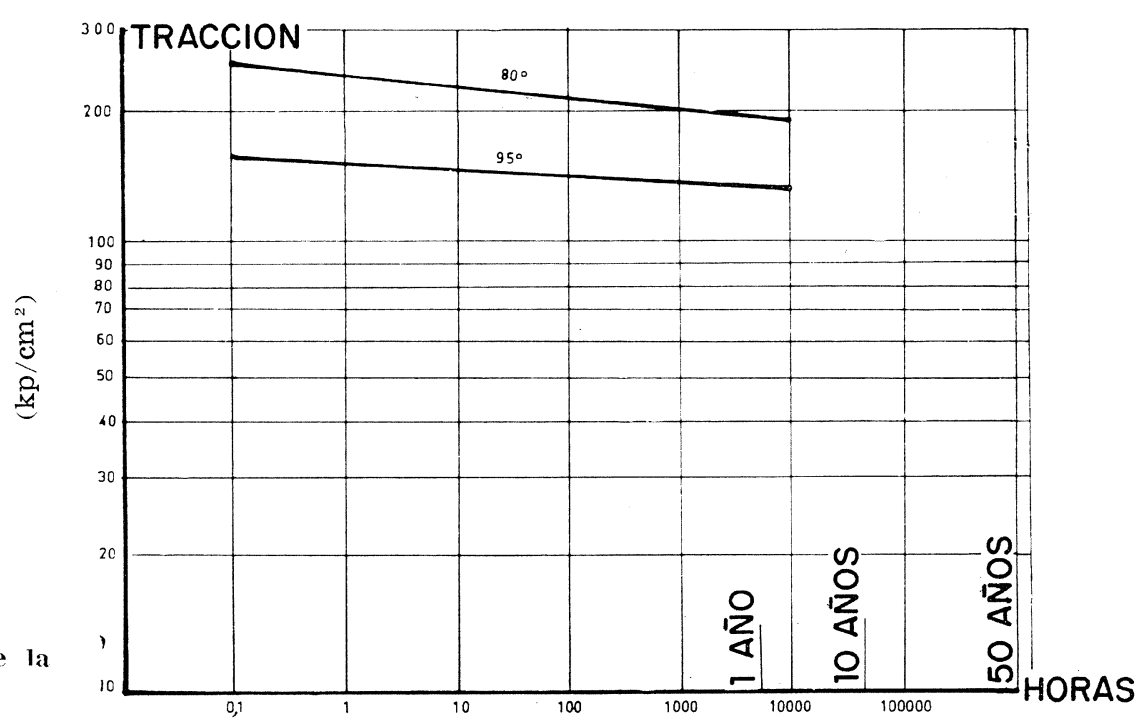

\section{Absorción de agua}

La absorción de agua puede limitar mucho la resistencia. Es sobre todo importante para los materiales plásticos termoendurecibles, que presentan a priori características térmicas y mecánicas muy interesantes. Para paliar este inconveniente, ciertos fabricantes revisten las paredes internas del tubo termoendurecible de una capa de P.V.C.: de este modo se obtiene un tubo de gran calidad, pero de un precio bastante poco competitivo.

\section{Coeficiente de dilatación}

Esta es una característica que diferencia mucho las materias plásticas de los metales, pues las dilataciones son de dos a diez veces más importantes (un tubo de $6 \mathrm{~m}$ puede dilatarse $4 \mathrm{~cm}$ bajo el efecto de una variación de temperatura de $50^{\circ} \mathrm{C}$ ). Si el instalador no tiene en cuenta este problema, los disgustos son irreparables. 


\section{Fragilidad}

Los tubos de material plástico son frágiles y más particularmente a baja temperatura. Conviene pues protegerlos contra los choques.

\section{Influencia en la potabilidad}

Ciertos materiales plásticos no pueden considerarse como adecuados en este aspecto. Esto se debe a veces a los productos de carga empleados como estabilizadores durante la fabricación.

\section{Inflamabilidad}

Excepto el P.V.C.C., los materiales plásticos considerados en este estudio son más o menos inflamables. Ciertos aditivos, como el cloro o el óxido de antimonio, mejoran la resistencia al fuego, pero en general en detrimento de las cualidades mecánicas y de envejecimiento.

\section{Retracción}

Ciertos tubos están sometidos a la retracción, por la relajación de tensiones internas impuestas por el método de fabricación. La retracción es más importante cuando el tubo está sometido al calor y se debe tener en cuenta en la puesta en obra.

\section{WECIO DE LOS TUBOS}

A sección de paso equivalente, el precio de los tubos de los materiales plásticos citados en esta nota, a excepción de los de poliamidas, es comparable al del acero (ver figura 6). Los racores, codos y juntas son, sin embargo, más costosos. Cuanto mayores son la temperatura y presión de utilización, los espesores del tubo de plástico deben ser más importantes. El precio de estos tubos es, pues, función de las condiciones de empleo: si son severas, la rentabilidad sólo puede compararse con la de los metales no férreos.

La ligereza permite ganar alrededor de un $20 \%$ sobre el transporte y la manutención de los tubos.

El costo de conservación es muy reducido gracias a la ausencia de corrosión e incrustaciones, a pesar de las deterioraciones por choque que corren el riesgo de ser más numerosas.

\section{CALCULO DE LOS TUBOS}

Los diámetros se calculan en función de los gastos y de las pérdidas de carga con ayuda de un ábaco especial (fig. 7). Este ábaco no está sin embargo admitido oficialmente.

El espesor de los tubos se calcula en función de la presión, del diámetro y de la resistencia del material, a partir de la fórmula siguiente:

$$
e=\frac{P D}{2 t}
$$


Fig. 6.-Precios comparados de los tubos.

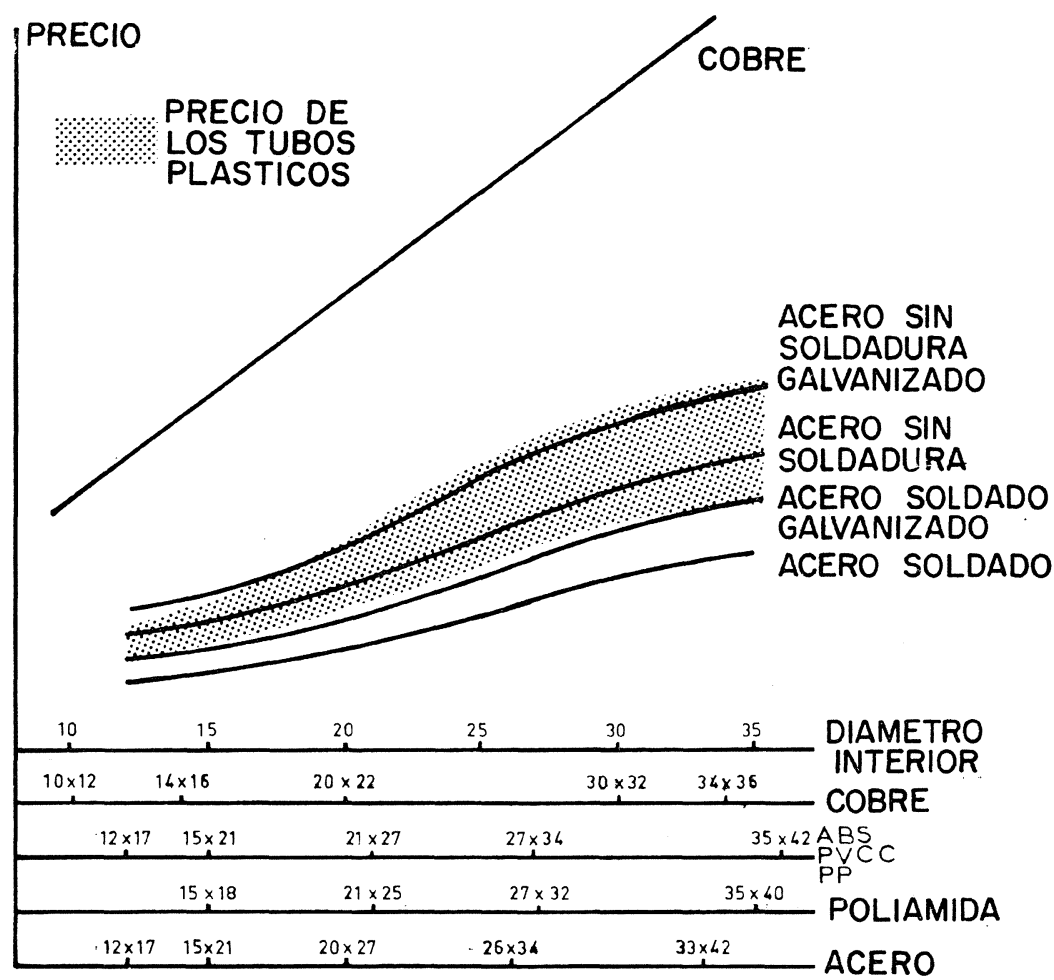

donde:

$e$ es el espesor del tubo (en $\mathrm{mm}$ );

$P$ es la presión de utilización $\left(e n \mathrm{kp} / \mathrm{cm}^{2}\right)$;

$D$ es el diámetro medio del tubo (en $\mathrm{mm}$ );

$t$ es la resistencia a tracción admisible para el material plástico (en $\mathrm{kp} / \mathrm{cm}^{2}$ ).

Esta tensión admisible es igual a la tensión de rotura a largo plazo del material, dividido por un coeficiente de seguridad.

La tensión de rotura depende, esencialmente, de la temperatura y de la duración del empleo del tubo. Es clásico tener en cuenta, como base de cálculo, la pérdida de resistencia después de 50 años de utilización. Como este valor sólo puede ser extrapolado, hay en esto una cierta incertidumbre. Generalmente, se representa la variación de la tensión de rotura en función del tiempo, según coordenadas logarítmicas. Los materiales plásticos que resisten bien a la temperatura aparecen bajo forma de rectas (fig. 8); los que son más sensibles a la temperatura se presentan bajo forma de curvas encorvadas (fig. 9), cuyo decrecimiento es más o menos importante. Para el P.V.C. rígido, la tensión de rotura a $20^{\circ} \mathrm{C}$, extrapolada a 50 años, se ha estimado en $75 \mathrm{kp} / \mathrm{cm}^{2}$. La tensión admisible fijada por la norma NFT 54003 es de $60 \mathrm{kp} / \mathrm{cm}^{2}$, así que el coeficiente de seguridad será de $75 / 60=1,25$. 
Fig. 7.-Determinación d e 1 diámetro interior de los tu-

bos de plástico (según los trabajos del profesor TISON, de la Universidaa de Garite).

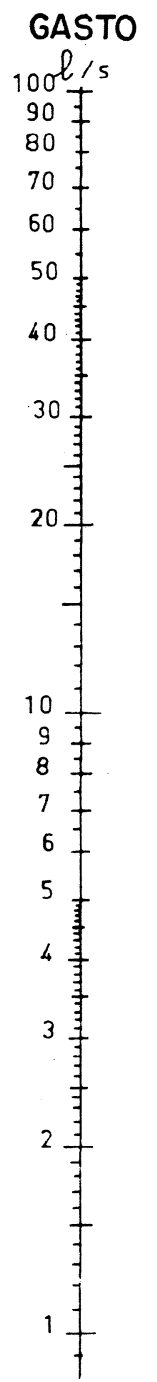

\section{DIAMETRO}

$\mathrm{mm}$

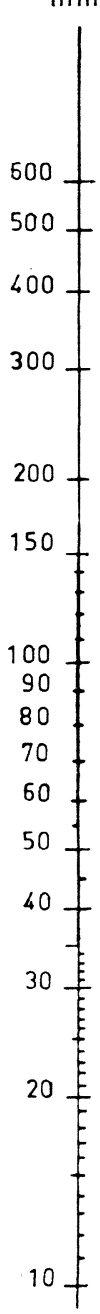

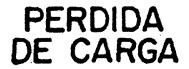

VELOCIDAD

MEDIA

$\mathrm{m} / \mathrm{s}$

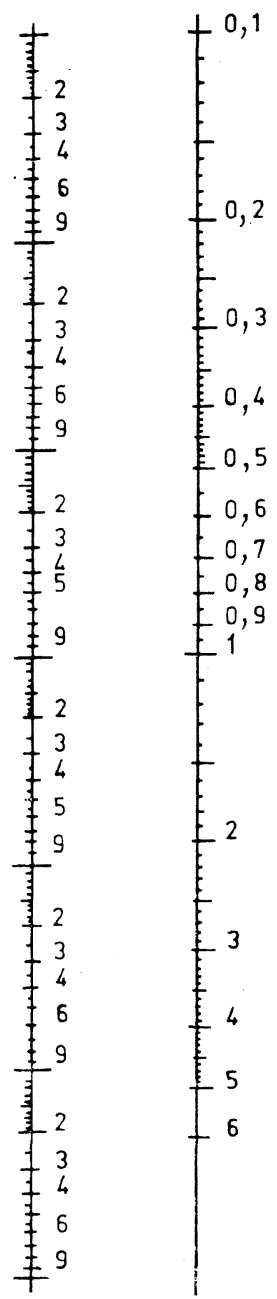

Para el P.V.C.C., el polipropileno y la poliamida, ciertos fabricantes aconsejan tomar las tensiones admisibles siguientes:

\begin{tabular}{|c|c|c|c|}
\hline \multirow{2}{*}{$\begin{array}{c}\text { Naturaleza } \\
\text { del tubo }\end{array}$} & \multicolumn{3}{|c|}{ Temperaturas de utilizacion } \\
\hline & $60^{\circ} \mathrm{C}$ & $80^{\circ} \mathrm{C}$ & $100^{\circ} \mathrm{C}$ \\
\hline P.V.C.C. & $50 \mathrm{kp} / \mathrm{cm}^{2}$ & $40 \mathrm{kp} / \mathrm{cm}^{2}$ & $12 \mathrm{kp} / \mathrm{cm}^{2}$ \\
\hline Polipropileno & $30 \mathrm{kp} / \mathrm{cm}^{2}$ & $25 \mathrm{kp} / \mathrm{cm}^{2}$ & $10 \mathrm{kp} / \mathrm{cm}^{2}$ \\
\hline Poliamida & $55 \mathrm{kp} / \mathrm{cm}^{2}$ & $38 \mathrm{kp} / \mathrm{cm}^{2}$ & $33 \mathrm{kp} / \mathrm{cm}^{2}$ \\
\hline
\end{tabular}

\section{PUESTA EN OBRA DE LOS TUBOS}

Ciertas prescripciones impuestas por las DTU son aplicables a todos los tubos. Sin embargo, según la naturaleza del plástico empleado, la puesta en obra puede crear proble- 
mas particulares. Por tanto es necesario remitirse a las indicaciones de los fabricantes.

El cimbreo en caliente presenta ciertos riesgos de ovalización o bien de fisuración; por eso es mejor emplear un codo para cada desviación. Los empalmes para el empotramiento pegado se hacen fácilmente ( $\sin$ embargo, ciertos materiales plásticos no se pueden pegar). Está prohibido el roscado, salvo si una disposición especial lo permite, siempre que no se debilite localmente el tubo; no se aconseja la soldadura en obra. Eventualmente, pueden realizarse los empalmes desmontables con bridas y cuellos o por engaste.

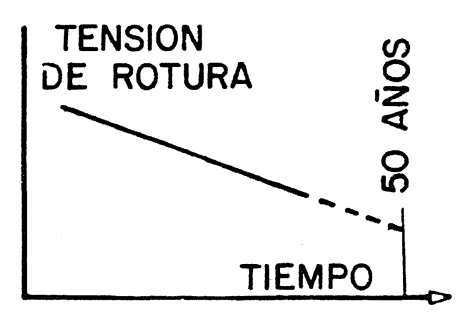

Fig. 8

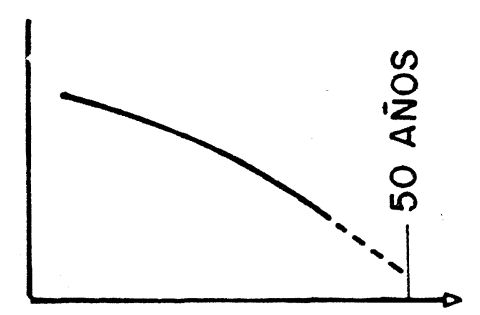

Fig. 9)

Los tubos se sujetan con collares ligeramente más grandes que el diámetro exterior, para permitir la libre dilatación del tubo. Sobre la horizontal, la separación de los collares es unas diez veces el diámetro exterior.

Las partes acodadas pueden absorber cierta dilatación, siempre que no estén apoyadas contra una pared o fijadas por un collar. Las partes rectilíneas deben ser interrumpidas cada $5 \mathrm{~m}$ aproximadamente por una junta corredera o por una lira. A la salida de cada acometida, es necesario disponer un codo para evitar la transmisión de los esfuerzos de dilatación (figs. 10 y 11).

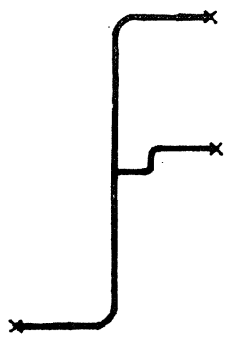

Fig. 10

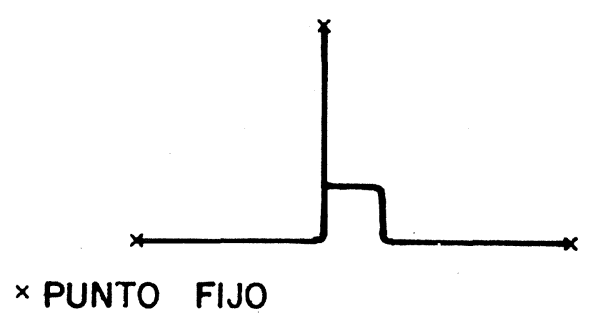

Fig. 11

\section{UE}

Esta breve vista panorámica de los materiales plásticos para canalizaciones de agua caliente es forzosamente aproximada, ya que, para un mismo material, la calidad difiere de un fabricante a otro, principalmente según la naturaleza y dosificación de los productos de carga. Permite al menos pulir los problemas y aportar algunas nociones de base a su utilización. 
De todas formas, cuando se considera la posibilidad de realizar una instalación con materias plásticas, es interesante asegurar la colaboración del fabricante y, eventualmente, realizar ensayos si las condiciones de servicio son muy severas.

En resumen, estos tubos son ya capaces de poderse emplear de modo rentable en muchos casos, pero su empleo no podrá generalizarse más que cuando las fabricaciones estén definitivamente a punto y las reglas de puesta en obra estén bien establecidas, para tener en clienta los problemas particulares que lleva consigo su utilización.

\section{TABLA COMPARATIVA DE LOS MATERIALES PLASTICOS}

\begin{tabular}{|c|c|c|c|c|c|}
\hline $\begin{array}{l}\text { Naturaleza } \\
\text { del M.op. }\end{array}$ & P.V.C.C. & A.B.S. & P.P. & Poliamida & $\begin{array}{l}\text { Termoendure- } \\
\text { cibles } \\
\text { estratificados }\end{array}$ \\
\hline $\begin{array}{l}\text { Comportamiento } \\
\text { al calor }\end{array}$ & Bueno & Bueno & Bueno & Bueno & Muy isueno \\
\hline Combustibilidad & No combustible & $\begin{array}{l}\text { Combustible, pe- } \\
\text { ro exist e en } \\
\text { autoextinguible }\end{array}$ & $\begin{array}{l}\text { Combustible, pe- } \\
\text { ro exist e en } \\
\text { autoextinguible }\end{array}$ & Autoextinguible & $\begin{array}{l}\text { Combustible, pe- } \\
\text { ro exis t e en } \\
\text { autoextinguible }\end{array}$ \\
\hline $\begin{array}{l}\text { R€sistencia quí- } \\
\text { mica }\end{array}$ & $\begin{array}{l}\text { Buena, pero sen- } \\
\text { sible a los disol- } \\
\text { ventes }\end{array}$ & $\begin{array}{l}\text { Buena, pero } \\
\text { sensible a los } \\
\text { ácidos oxidantes }\end{array}$ & $\begin{array}{l}\text { Buena, pero } \\
\text { sensible a los } \\
\text { ácidos oxidantes }\end{array}$ & Buena & Buena \\
\hline Envejecimiento & Bueno & Medio & Bueno & Bueno & Bueno \\
\hline Cimbreo & Delicado & Fácil & Fácil & F'ácil & Imposible \\
\hline $\begin{array}{l}\text { Coeficiente de } \\
\text { dilatación }\end{array}$ & $7 \times 10^{-5} / 0 \mathrm{C}$ & $8 \times 10^{-5}$ & $12 \times 10^{-5}$ & $15 \times 10^{-5}$ & $3 \times 10^{-5}$ \\
\hline Ensamblado & Fácil & Fácil & $\begin{array}{l}\text { Pegado imposi- } \\
\text { bie }\end{array}$ & Fácil & Difícil \\
\hline Densidad & 1,5 & 1,1 & 0,9 & 1,04 & 1,6 \\
\hline $\begin{array}{l}\text { Temperatura Vi- } \\
\text { cat bajo carga } \\
\text { de } 5 \mathrm{kp}\end{array}$ & $110^{\circ} \mathrm{C}$ & - & $100^{\circ} \mathrm{C}$ & $160^{\circ} \mathrm{C}$ & - \\
\hline
\end{tabular}

